\title{
Avaliação do ESTADO NUTRIIIONAL dE CRIANÇAS E ADOLESCENTES COM INSUFIIIENCIA RENAL CRÔNICA
}

\author{
M.P. Zambon*, V.M.S. Belangero, A.C.G. de Britto, A.M. Morcillo \\ Trabalhorealizadona UniversidadeEstadual de Campinas-Hospital das Clínicas daUnicampeCiped- \\ Centro deinvestigaçõesemPediatria daUnicamp. FaculdadedeCiências Médicas-Departamento dePediatria, Campinas, SP.
}

RESUMO - A desnutrição está freqüentemente relacionada com doenças crônicas. 0 diagnóstico precoce realizado por técnicas práticas e simples favorece 0 tratamento, minimizando as complicaçōes. Objetivo: avaliar a situação nutricional de crianças e adolescentes com insuficiência renal crônica (IRC) utilizando parâmetros antropométricos.

Métodos. Foram avaliadas 4 I crianças e adolescentes com IRC ("clearance" de creatinina abaixo de $50 \mathrm{~m} / / \mathrm{min} / 1,73 \mathrm{~m} 2$ ) acompanhadas no Ambulatório de Nefrologia Pediátrica do HC-Unicamp, de janeiro de 1995 a novembro de 1996, pelas medidas do perímetro braquial (PB) e da prega cutânea tricipital (PCT), com as quais calculou-se a área de gordura braquial (AGB) e área muscular braquial (AMB) e os respectivos escores $z$. Estas medidas foram realizadas pelo menos duas vezes, com intervalo de $0,2 \mathrm{I}$ a $\mathrm{I}, 3$ anos $(0,88 \pm 0,04)$.
Resultados. Os escores $z$ obtidos tanto para PB, como para PCT, AGB e AMB apresentaram valores muito baixos, sendo que, na evolução, apenas 0 escore $z$ de $A M B$ apresentou melhora estatisticamente significativa ( $p=0,03$ teste de Wilcoxon). Quando selecionados quanto ao tratamento de substituição renal, apenas os transplantados renais apresentaram melhora estatisticamente significativa para 0 escore $\mathbf{z}$ de $\mathrm{PB}(\mathrm{p}=0,02$ teste de Wilcoxon).

Conclusão. Estes dados mostram uma situação nutricional comprometida tanto em relação à reserva de gordura, quanto de músculo, com melhora no acompanhamento apenas em relação à área muscular.

UnITERMOS: Insuficiência renal crônica. Avaliação nutricional. Antropometria.

\section{INTRODUÇÃO}

A desnutrição energético-protéica está freqüentemente associada às doenças crônicas. Anteriormente, não era considerada um problema prioritário, porém o reconhecimento precoce, com rápida intervenção no suporte nutricional, permite minimizar o impacto, levando a benefícios clínicos, mesmo na ausência de um efetivo tratamento da doença de base.

Para a avaliação nutricional podem ser utilizados métodos clínicos, laboratoriais (albumina, transferrina, pré-albumina, contagem de linfócitos, etc) e antropométricos. Os dados clínicos são pouco úteis devido a

\footnotetext{
*Correspondência:Mariana PortoZambon Rua: Botafogo, 151/491 Caminhos de San Conrado Sousas-Campinas-São Paulo- Brasil CEP: 13130-601 Fax:(019)788-8260-Email:zambon@correionet.com.br
}

grande variação na forma e freqüência de aparecimento, aliado ao fato de estarem presentes somente nas formas moderadas e graves. Quanto aos parâmetros laboratoriais citados, as alterações também ocorrem apenas nas formas moderadas e graves da desnutrição, necessitam da coleta de material (sangue) e de laboratórios de boa qualidade, o que acarreta aumento no custo operacional.

Neste ponto, a antropometria apresenta vantagens, pois não é invasiva, tem baixo custo e permite o diagnóstico precoce. As medidas mais utilizadas são o peso e a altura, sendo que a relação peso/idade e peso/altura são indicadores da situação nutricional atual do paciente, enquanto a altura/idade está relacionada ao passado nutricional. O peso, que expressa a massa corporal total, pode sofrer variações em curtos intervalos de tempo. Pouca informação nos dá sobre a composição corporal e seu emprego é discutível na avaliação de pacientes com algumas doenças crônicas nas quais ocorre retenção hídrica!.

Atualmente, vários métodos têm sido empregados para estudar a composição corporal, destacando-se a determinação da densidade corporal a partir do peso submerso, a diluição de radioisótopos, a tomografia computadorizada, a ressonância nuclear magnética, a DEXA (Dual-Energy $X$ Ray absorptiometry), a bioimpedância e a antropometria (pregas cutâneas, perímetros, etc), todos com o propósito de estimar, indiretamente, a gordura corporal total e a massa corporal livre de gordura ${ }^{2-5}$. Alguns são agressivos, com alto custo e 
inconvenientes para o paciente; outros carecem de estudos de validação que permitam o uso em crianças, em geral de pouca aplicação clínica.

A partir do perímetro braquial (PB) e da prega cutânea tricipital (PCT), pode-se calcular as áreas de gordura (AGB) e muscular braquial (AMB) 6,7 . A PCT é um indicador da gordura corporal, enquanto as medidas de PMB e AMB se correlacionam com a massa muscular 6 . A desnutrição causa diminuição na gordura e no músculo do braço, que pode ser facilmente avaliada pelas medidas braquiais 8,9 .

$\mathrm{Na}$ IRC, a desnutriçãa, complicação freqüente, deve ser precocemente diagnosticada e corrigida. Blumenkrantz et al. (1980) ( I0) sugerem, para sua detecção, o uso da $P C T, P M B$ e $A M B$, por serem de simples aplicação e suficientes para os propósitos clínicos.

O objetivo deste trabalho foi avaliar o estado nutricional de crianças e adolescentes do Ambulatório de Nefrologia Pediátrica do Hospital das Clínicas da Unicamp, empregando o PB, a PCT, a AGB e a AMB.

\section{Métodos}

Realizou-se um estudo transversal que incluiu 4 I crianças e adolescentes do Ambulatório de Nefrologia Pediátrica do Hospital das Clínicas da Unicamp, com diagnóstico de IRC ("clearance" de creatinina inferior a $50 \mathrm{ml} / \mathrm{min} / \mathrm{l}, 73 \mathrm{~m} 2$ ) entre janeiro de 1995 e novembro de 1996.

O "clearance de creatinina" foi realizado com coleta de urina de 24 horas. Após a medida do volume, a urina foi homogeneizada e uma alíquota foi diluída a I/I0, sendo a dosagem de creatinina sérica e urinária realizada de forma automática no aparelho COBAS MIRA.

Os dados referentes ao sexo, idade, idade de início da IRC, tempo de IRC, etiologia da insuficiência renal, tratamento

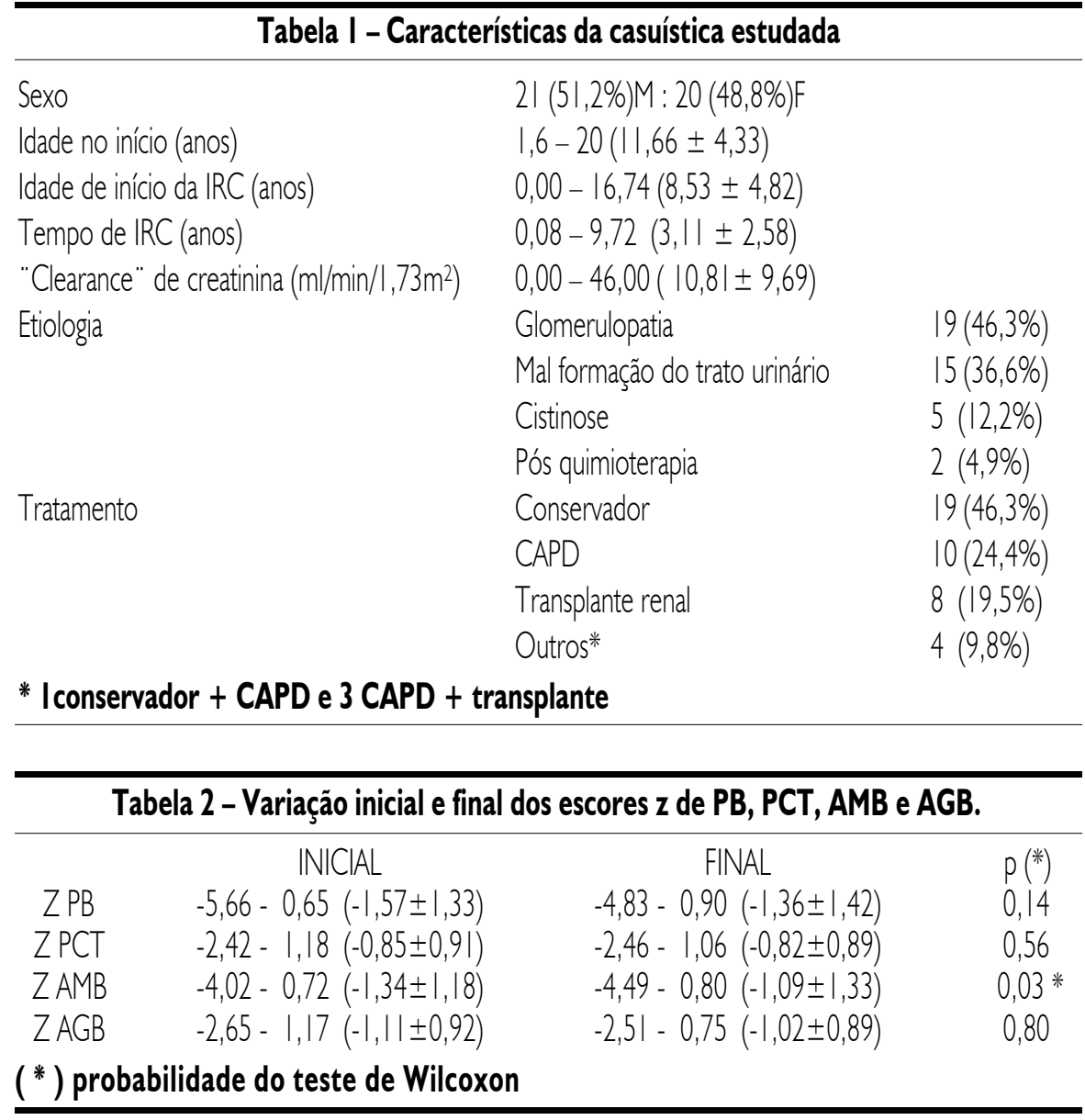

atual e "clearance" de creatinina são apresentados na Tabela I.

As crianças foram submetidas a um exame antropométrico realizado por um dos autores, que incluiu as medidas do PB e da PCT pelas técnicas propostas por Mascarenhas ${ }^{6}$. Com estas medidas, calculou-se a $A M B$ e a $A G B$ e os escores $z$ de PB, PCT, $A M B$ e $A G B$, empregando os dados de referência de Frisancho ${ }^{7}$. Nenhum dos pacientes analisados apresentava edema clinicamente evidente. $\mathrm{O}$ exame antropométrico foi realizado, no mínimo, duas vezes em cada paciente, com intervalo de 0,21 a 1,3 anos $(0,88 \pm 0,04)$.

Os pacientes foram divididos em quatro grupos, segundo a etiologia da IRC e ao tratamento utilizado durante o período de estudo. Etiologia: I- todas as glomerulopa- tias primárias e as secundárias às doenças sistêmicas; I- as hipoplasias, displasias e malformações do trato urinário com ou sem infecção de repetição; III- as doenças metabólicas genéticas não glomerulares (cistinose); IV- a IRC resultante de quimioterápicos. Tratamento: I- conservador, IICAPD, III- transplante renal, IV- alteração no tipo de tratamento durante o período de avaliação (conservador para CAPD ou CAPD para transplante).

$\mathrm{Na}$ análise dos dados, utilizou-se o programa SPSS. Os escores z de PB, PCT, AMB e AGB foram comparados entre as duas avaliações para o conjunto dos pacientes e em relação aos tipos de tratamento.

As variáveis contínuas são apresentadas em tabelas, contendo seus valores extremos, a média e o desvio padrão $(m \pm D P)$. 
Tabela 3 - Variação inicial e final dos escores z de PB, PCT, AMB e AGB, de acordo com o tratamento.

\begin{tabular}{|c|c|c|c|c|c|c|c|c|c|c|c|c|}
\hline & \multicolumn{3}{|c|}{$\begin{array}{c}\text { CONSERVADOR } \\
(n=19)\end{array}$} & \multicolumn{3}{|c|}{$\begin{array}{l}\text { CAPD } \\
(n=10)\end{array}$} & \multicolumn{3}{|c|}{$\begin{array}{l}\text { TRANSPLANTE } \\
(\mathrm{n}=8)\end{array}$} & \multicolumn{3}{|c|}{$\begin{array}{l}\text { MUDANÇA DE TRATAMENTO } \\
(n=4)\end{array}$} \\
\hline & INICIAL & FINAL & $\mathbf{p}(*)$ & INICIAL & FINAL & $\mathrm{p}(*)$ & INICIAL & FINAL & $\mathrm{p}(*)$ & INICIAL & FINAL & $\mathbf{p}(*)$ \\
\hline ZPB & $\begin{array}{l}-4,25 \text { a } 0,43 \\
(-1,55 \pm 1,31)\end{array}$ & $\begin{array}{c}-4,7 \text { a } 0,35 \\
(-1,46 \pm 1,22)\end{array}$ & 0,96 & $\begin{array}{l}-5,66 \text { a } 0,60 \\
(-1,49 \pm 1,73)\end{array}$ & $\begin{array}{l}-4,83 \text { a } 0,90 \\
(-1,60 \pm 1,89)\end{array}$ & 0,64 & $\begin{array}{l}-2,48 \text { a } 0,65 \\
(-1,33 \pm 1,06)\end{array}$ & $\begin{array}{l}-1,79 a a, 54 \\
(-0,76 \pm 0,81)\end{array}$ & $0,02 *$ & $\begin{array}{l}-3,68 \mathrm{a}-1,48 \\
(-2,27 \pm 1,03)\end{array}$ & $\begin{array}{l}-4,40 \text { a } 0,18 \\
(-1,50 \pm 2,10)\end{array}$ & 0,27 \\
\hline ZPCT & $\begin{array}{l}-1,97 \text { a } 0,38 \\
(-0,75 \pm 0,69)\end{array}$ & $\begin{array}{l}-2,22 \text { a } 0,02 \\
(-0,94 \pm 0,64)\end{array}$ & 0,05 & $\begin{array}{l}-1,77 \text { a } 1,18 \\
(-0,77 \pm 1,02)\end{array}$ & $\begin{array}{l}-2,05 \text { a } 0,83 \\
(-0,78 \pm 1,05)\end{array}$ & 0,72 & $\begin{array}{l}-2,36 \text { a } 0,94 \\
(-0,71 \pm 1,24)\end{array}$ & $\begin{array}{l}-2,46 \text { a } 1,06 \\
(-0,43 \pm 1,17)\end{array}$ & 1,00 & $\begin{array}{l}-2,42 \text { a } 1,39 \\
(-1,78 \pm 0,46)\end{array}$ & $\begin{array}{l}-2,12 \text { a } 0,19 \\
(-1,13 \pm 0,96)\end{array}$ & 0,10 \\
\hline ZAMB & $\begin{array}{l}-3,47 a 0,63 \\
(-1,35 \pm 1,31)\end{array}$ & $\begin{array}{l}-4,49 \text { a } 0,80 \\
(-1,14 \pm 1,32)\end{array}$ & 0,17 & $\begin{array}{l}-4,02 \text { a } 0,43 \\
(-1,20 \pm 1,27)\end{array}$ & $\begin{array}{l}-4,08 \text { a } 0,06 \\
(-1,34 \pm 1,64)\end{array}$ & 0,57 & $\begin{array}{l}-2,53 \text { a } 0,72 \\
(-1,36 \pm 0,99)\end{array}$ & $\begin{array}{l}-2,62 \mathrm{a} 0,64 \\
(-0,83 \pm 0,97)\end{array}$ & 0,09 & $\begin{array}{l}-3,03 \text { a } 0,91 \\
(-1,57 \pm 0,97)\end{array}$ & $\begin{array}{l}-3,09 \text { a } 0,47 \\
(-0,83 \pm 1,56)\end{array}$ & 0,14 \\
\hline ZAGB & $\begin{array}{l}-2,43 \text { a } 0,21 \\
(-1,03 \pm 0,66)\end{array}$ & $\begin{array}{l}-2,02 \text { a } 0,10 \\
(-1,15 \pm 0,65)\end{array}$ & 0,17 & $\begin{array}{l}-2,22 \text { a } 1,17 \\
(-1,03 \pm 1,12)\end{array}$ & $\begin{array}{l}-2,51 \text { a } 0,75 \\
(-1,02 \pm 1, \mid I)\end{array}$ & 0,95 & $\begin{array}{l}-2,64 \text { a } 0,48 \\
(-0,94 \pm 1,20)\end{array}$ & $\begin{array}{l}-2,38 \text { a } 0,69 \\
(-0,61 \pm 1,01)\end{array}$ & 0,48 & $\begin{array}{l}-2,65 \text { a I, } 46 \\
(-2,05 \pm 0,63)\end{array}$ & $\begin{array}{l}-2,30 \text { a } 0,22 \\
(-1,21 \pm 1,13)\end{array}$ & \\
\hline
\end{tabular}

Utilizou-se, para as variáveis discretas, a freqüência absoluta (N) e a relativa (\%). $\mathrm{Na}$ análise estatística utilizou-se o teste de Wilcoxon. Adotou-se o nível de 5\%, para excluir a hipótese de nulidade.

\section{Resultados}

Os escores z do PB da avaliação inicial variaram de $-5,66-0,65(-1,57 \pm 1,33)$ e na final $-4,83-0,90(-1,36 \pm 1,42)$. Para a PCT, a variação foi de $-2,42-1,19(-0,85$ $\pm 0,92)$ na inicial e $-2,46-1,07(-0,83 \pm$ $0,89)$ na final. Em relação a $A G B$, os escores $z$ iniciais variaram de $-2,65-1,17(-1,12 \pm$ $0,93)$ e os finais de $-2,51-0,75(-1,02 \pm$ $0,89)$. Os escores $z$ da AMB, inicial e final, variaram, respectivamente, de $-4,02-0,73$ $(-1,34 \pm 1,18)$ e $-4,49-0,80(-1,10 \pm$ I,33). A comparação entre os escores $z$, iniciais e finais, de cada parâmetro antropométrico, apresentou diferença estatisticamente significativa apenas para a AMB. A distribuição dos dados é apresentada na Tabela 2.

A distribuição dos escores z no início e no final do período de observação, em relação a cada grupo de tratamento, é apresentada na Tabela 3. Observou-se diferença estatisticamente significativa apenas no escore $z$ de PB no grupo transplantado.

\section{Discussão}

A desnutrição energético-protéica é muito freqüente em pacientes com IRC. Entre as múltiplas causas destacam-se a ingestão deficiente de nutrientes, perda protéica nos processos dialíticos, distúrbios endocrinológicos e do equilíbrio ácido-básico. A desnutrição destes pacientes pode piorar a evolução clínica pois favorece quadros infecciosos, dificulta a reabilitação do paciente e piora sua qualidade de vida. Portanto, tornam-se necessários métodos diagnósticos que associem precisão e fácil aplicação à rotina da clínica diária para que se possa atuar rapidamente.

Os indicadores braquiais de gordura e massa muscular já vêm sendo utilizados há algum tempo para determinação do estado nutricional, sendo a diminuição dos depósitos de gordura comum na região tricipital em crianças cronicamente desnutridas 6 .

Neste estudo, observou-se grande diminuição da PCT, da AGB e da $A M B$ no início do acompanhamento, demonstrando comprometimento das reservas de energia e de proteínas. A análise dos dados, após o período de observação, demonstrou que não houve mudança nos escores z da PCT e da AGB; ocorreu sim, um aumento na $A M B$, indicando que estas crianças, independente do tipo de trata- mento, não tiveram piora da situação nutricional ao longo do período do estudo.

$\mathrm{Na}$ literatura, os dados sobre IRC e desnutrição são escassos. lanhez et al. (1983) " estudaram 16 adultos em hemodiálise crônica e analisaram o peso, o PB e as pregas tricipital, subescapular, abdominal e de crista ilíaca, além das dosagens séricas de uréia, creatinina e albumina e também não demonstraram piora do estado nutricional dos pacientes quando bem conduzidos. Thunberg et al. ( I 98 I) (I2) estudaram 58 pacientes em esquema de hemodiálise e encontraram a PCT diminuída em $72 \%$, sendo a relação peso/altura, o PB e as dosagens séricas de albumina praticamente normais.

Por outro lado, Cuppari et al. ( 1989$)^{13}$, avaliando o estado nutricional de cem pacientes, de dez centros de hemodiálise de São Paulo, utilizando o inquérito nutricional e a análise antropométrica, não observaram alteração do peso dos pacientes, porém a PCT estava diminuída, principalmente nas mulheres (69\%) e um PB baixo, principalmente nos homens (79\%), sendo que ambos os parâmetros pioraram com a hemodiálise. Estes dados são semelhantes aos encontrados por Schoenfeld (1983) $)^{14} \mathrm{em}$ estudo cooperativo norte-americano.

Neste estudo, não dispomos de informações sobre a dieta destes pacientes, as- 
pecto que seria muito relevante no planejamento da recuperação nutricional, pois de acordo com Thunberg et al. (198I) $)^{12}$, Schoenfeld et al. (1983)14 e Cuppari et al. $(1989)^{13}$ seria a baixa ingesta a principal causa da desnutrição.

\section{Conclusão}

Os valores extremamente diminuídos dos parâmetros nutricionais estudados demonstram um comprometimento nutricional, tanto em relação à reserva de gordura, quanto de músculo, com melhora durante o acompanhamento, apenas em relação à área muscular, o que salienta a necessidade de um diagnóstico mais precoce da desnutrição em crianças e adolescentes com IRC e uma abordagem nutricional prioritária na condução destes casos.

Este diagnóstico precoce pode ser realizado periodicamente pelas técnicas antropométricas (PB e PCT), que, associadas a avaliação sistemática da composição da dieta e a utilização de um parâmetro laboratorial como a pré-albumina na rotina de atendimento destes pacientes, propiciaria valiosas informações para o tratamento e recuperação.

\section{SUMMARY}

\footnotetext{
NUTRITIONAL STATUS OF CHILDREN AND TEENAGERS WITH CHRONIC RENAL FAILURE

BACKGROUND. the purpose of this study is to evaluate the nutritional status of children and teenagers with chronic renal (CRF) failure
}

using anthropometric measurements.

Material and methods. 4 I children and adolescents with (CRF) (creatinine "clearance" $<50 \mathrm{~m} / \mathrm{min} / \mathrm{l}, 73 \mathrm{~m} 2$ ) followed at the Pediatric Nephrology Unit (HC-UNICAMP), between January 1995 and November 1996, were evaluated by the assessment of anthropometric measurements, mid upper arm circunference (MUAC) and triceps skinfold (TSF). With these measurements, mid arm fat area (MUAFA) and arm muscle area (AMA) were calculated and its z-scores. These measurements were performed twice at least, ranging from 0.21 to 1.3 years $(0.88$ \pm 0.04 ).

RESULTS, all the z-scores (MUAC, TSF, MUAFA and AMA) were very low, and only the AMA z-score was statistically significant ( $p=0,03$ Wilcoxon test). The patients were divided in to groups, according to their treatment, and the MAC $z$-score for the renal transplantation group was statistically significant ( $p=$ 0,02 Wilcoxon test).

Conclusions. these data demonstrate a compromised nutritional status from both muscle and fat stores, with an improvement in muscle stores. [Rev Ass Med Brasil 200 I; 47(2): 137-40]

KEY WordS: Chronic renal failure. Nutritional evaluation. Anthropometry.

\section{REFERÊNCIAS}

I. Frisancho AR. Anthropometric Standads for the Assessment of Growth and Nutritional
Status. Ann Arbor, The University of Michigan Press, 1993. p. 189.

2. Lohman TG. Skinfolds and Body Density and Their Relation to Body Fatness: A Review. Human Biology. 1981;53:181-225.

3. Deurenberg P. Methods for determining fat mass and fat distribution. Acta Paediatr (Suppl) 1992; 383:53-7.

4. Lukaski HC. Methods for the assessment of human body composition: tradicional and new. Am I Clin Nutr. 1987:46:537-56.

5. Katch FI, Katch VL. Avaliação da Composição Corporal. In: Katch FI, Katch VL. Fisiologia do Exercício. Energia, nutrição e desempenho humano. $4^{\circ}$ ed, Rio de Janeiro, Guanabara Koogan, 1998;5।3-547.

6. Mascarenhas MR, Zemel B, Stallings VA. Nutritional Assessment in Pediatrics. Nutrition 1998; | 4: I05- I 5.

7. Frisancho AR. New norms of upper limb fat and muscle areas for assessment of nutritional status. Am J Clin Nutr. I 98I;34:2540-5

8. Jelliffe EFP, Jelliffe DB. The arm circumference as a public health index of proteincalorie malnutrition of early childhood. J Trop Pediatr. 1969; 15:179-88.

9. Jelliffe DB, Jelliffe EFP. The arm circumference as a public health index of protein-calorie malnutrition of early childhood. J Trop Pediatr. 1969; 1 5:253-60.

I0. Blumenkrantz MJ, Kopple JD, Gutman RA etal. Methods for assessing nutritional status of patients with renal failure. Am J Clin Nutr. 1980; 33:1567-85.

I I. lanhez LZ, Jota de Paula F, Sabbaga E. Avaliação do Estado Nutricional de Pacientes Urêmicos em hemodiálise crônica. J Bras Nefrol. 1983:5:43-6.

12. Thunberg BJ, Swamy AP, Cestero RVM. Crosssectional and longitudinal nutritional measurements in maintenance hemodialysis patients. Am J Clin Nutr. 1981;34:2005- 12.

13. Cuppari L, Draibe AS, Anção MS et al. Avaliação Nutricional em Pacientes Renais Crônicos em Programa de Hemodiálise. Estudo Multicêntrico. Rev Ass Med Brasil. 1989; 35:9-14.

14. Schoenfeld PY, Henry RR, Laird NM, Roxe, DM. Assessment of nutritional status of the National Cooperative Dialysis Study population. Kidney Int (Suppl I 3). 1983;23:80-8. 